Original article

\title{
Clinical profile and role of serology in pediatric acute febrile illness: Experience from a tertiary care hospital in South India
}

\author{
Vaishnavi Kavirayani $^{a}$, Mridula Madiyal ${ }^{\text {b, }}$, Shrikiran Aroor $^{c}$, Sonakshi Chhabra ${ }^{a}$ \\ ${ }^{a}$ Kasturba Medical College, Manipal, Manipal Academy of Higher Education, Manipal, Karnataka - 576104, India \\ ${ }^{\mathrm{b}}$ Department of Microbiology, Kasturba Medical College, Manipal, Manipal Academy of Higher Education, Manipal, Karnataka - 576104, India \\ ${ }^{\mathrm{c}}$ Department of Pediatrics, Kasturba Medical College, Manipal, Manipal Academy of Higher Education, Manipal, Karnataka - 576104, India
}

\section{A R T I C L E I N F O}

\section{Keywords:}

Acute febrile illness

Pediatric AFI

Serology

Fever

Percentage positivity

\begin{abstract}
A B S T R A C T
Problem addressed: Acute febrile illness (AFI) is a diagnostic challenge for the laboratory and clinicians. Serological diagnosis plays a major role in diagnosis of AFI. The data regarding pediatric AFI is limited. The present study was conducted to evaluate the clinical spectrum of acute febrile illness (AFI) in pediatric patients, along with the role of various diagnostic modalities.

Method: It was a retrospective observational study. The clinical and laboratory data for the pediatric AFI cases were collected and analyzed for percentage of etiologies and role of serological assays.

Results: Out of 214 cases studied, $57.9 \%$ were males, and $42.1 \%$ were females. Majority children were $<10$ years. Fever alone was seen in $36 \%$ of cases. The other common presentations were upper respiratory tract illness and gastroenteritis. Mean duration of fever was six days. Leukocytosis was seen in $36.4 \%$ of cases. Increased AST and ALT was noted in $48.7 \%$ and $39.5 \%$ of cases. Serological tests gave a positive result in $14.9 \%$ of cases. Most common disease diagnosed was Dengue (10/94 samples tested). Percentage positivity for different immunoassays was 31.8\% for Hepatitis A- ELISA, 9\% for Hepatitis E ELISA, 2.9\% for Scrub typhus ELISA, 5\% for Leptospirosis ELISA, $10.6 \%$ for Dengue ELISA. Febrile agglutination tests for Brucellosis, Typhoid and Rickettsial diseases showed positivity of 3.9\%, 1.7\% and 7.1\% respectively. Clinical recovery was documented in $96.5 \%$ of cases with no significant association with serological positivity.

Conclusion: Serology is important in diagnostic work-up of AFI in children. However, the approach towards diagnosis needs to be syndromic with other modalities depending on the clinical suspicion.
\end{abstract}

\section{Introduction}

Fever is known to be the most common symptom in any systemic illness and has a huge role to play in morbidity. Every year, different parts of the world are affected by seasonal fevers. ${ }^{1,2}$

Acute febrile illness (AFI) is classically defined as fever $<2$ weeks, rapid onset, caused by diverse pathogens without any system or organspecific aetiology. Due to overlapping clinical presentation and limited microbiological services available in many low resource areas, very few patients receive an accurate and specific diagnosis at the community level, the relative importance of various causes of fever remains unknown.

Fever in children signifies systemic inflammation, typically in response to a viral, bacterial, parasitic, or less commonly, a noninfectious aetiology. Geographic settings and patients' ages can help point to the appropriate diagnosis and treatment if local epidemiology is well understood. AFI can be potentially fatal if the aetiology is not recognised and if not appropriately treated early.

Mostly seen in the tropical and subtropical regions, AFI has the highest incidence in childhood-majority between 4 and 5 years of age. In tropical countries like India, the most common reasons for AFI are Dengue, Malaria, Typhoid fever, Leptospirosis and Rickettsial infection. Acute respiratory infections and viral exanthematous fevers also affect children significantly. Population density and urbanisation may contribute to the emergence and re-emergence of some of these diseases in tropical regions. ${ }^{3}$

\footnotetext{
* Corresponding author. Department of Microbiology, Kasturba Medical College, Manipal, Manipal Academy of Higher Education, Manipal, Karnataka - 576104, India.

E-mail addresses: vaishnavi.kavirayani@learner.manipal.edu (V. Kavirayani), mridula.m@manipal.edu (M. Madiyal), shrikiran.a@manipal.edu (S. Aroor), Sonakshi.chhabra29@gmail.com (S. Chhabra).
} 
In tropical countries, malaria is frequently the cause of AFI followed by other non-malarial reasons. ${ }^{4-6}$ Viral respiratory tract infections are more seen in middle east countries.

The most critical issue for primary-care physicians is to focus on the fever's aetiology and to rule out serious diseases. Distinguishing a patient with viral illness from one with bacterial infection is essential as there may be considerable overlap in the clinical appearance.

Due to uncertainties and shortage of data in this field, clinical decision-making often gets compromised. There is deficiency of data in pediatric age group regarding AFI. Understanding the pattern of AFI and decoding various clinical and diagnostic clues will help to identify the aetiology, thereby expediting the treatment process. In this context, we have conducted a cross-sectional retrospective study to determine the prevalence of local infectious diseases causing AFI in our geographical area. The objectives were to evaluate the microbiological aetiology of acute febrile illness (AFI) in pediatric patients using serological and bacterial cultures and to understand the clinical profile of acute febrile illness in pediatric patients.

\section{Materials and methods}

A cross-sectional retrospective 6-month study was conducted in a tertiary health-care hospital of South India (Karnataka) from January 2018 to June 2018.

\subsection{Search strategy}

The research included pediatric patients of age group $<18$ years, admitted to the hospital with febrile illness between the 6-month period of study. The study was approved by the institutional ethics board. Ethic clearance number: IEC 505-2019.

Hospital numbers of pediatric patients were collected and evaluated for AFI from microbiology laboratory records. Clinical profile of each patient, which includes history of illness, symptoms, signs, investigations undertaken and respective management was documented from hospital records as per the proforma.

\subsection{Information and data extraction}

The proforma constituted general information of the patient (age, gender, residence, date of admission, date of discharge, etc.), associated risk factors (like undernutrition, immunosuppression, intake of poultry/ meat, contact with domestic animals, history of insect bite, etc.), associated organ systems that were particularly involved, clinical symptoms and signs - with duration and detailed description of each and laboratory diagnosis, which involved biochemical, hematological, microbiological, serological and statistical analysis.

\subsection{Laboratory diagnosis}

The biochemical and hematological parameters mainly included liver function markers, blood indices and inflammatory markers. The data collected also included microbiological work up such as immunoassay, molecular tests, and bacterial cultures. The serological investigations used for analysis were commercial Enzyme Linked Immunosorbent assays (ELISA) for the detection of IgM antibodies of the various infectious etiologies for AFI. The list included IgM ELISA result for Scrub typhus, Leptospirosis, Hepatitis A, Hepatitis E and Dengue. We also collected results of febrile agglutination tests as Widal test, Weil Felix test and Standard agglutination test for Brucellosis.

\subsection{Statistical analysis}

The statistical analysis mainly involved calculation of percentages. Categorical variables have been expressed in percentages and continuous variables in median and interquartile range. Chi-square test was used to find the association between categorical variables. The result was considered statistically significant when p-value obtained was $<0.05$.

\section{Results}

357 pediatric AFI patients were evaluated, who visited the hospital between January 2018 to June 2018. The study team could retrieve clinical data for 214 cases. Out of 214 patients, 124 (57.9\%) were males, and $90(42.1 \%)$ were females. Nearly $34.1 \%$ were $<5$ years of age, $33.2 \%$ belonged to $6-10$ years of age, $25.2 \%$ to $11-15$ years of age and the least, $7.5 \%$ to age group $>15$ years [Table 1]. The mean affected age was $8.23 \pm 4.08$ years and average duration of fever at the time of presentation was six days.

All the patients showed fever as the primary symptom. Seventyseven cases accounting for $36 \%$ presented with fever alone, whereas other patients had symptoms persistent to various organ systems. Upper respiratory tract and gastrointestinal tracts are the frequently involved systems with $19.6 \%$ and $18.2 \%$ respectively. Twenty-five cases (11.6\%) presented with exanthematous fever. Documentation of undernourishment was noted in $50 \%$ of the patients, whereas, $19 \%$ were immunosuppressed, $1.7 \%$ of the patients consumed raw milk, and were in recent

Table 1

Demographic Characteristics in pediatric patients suffering from AFI.

\begin{tabular}{|c|c|c|c|}
\hline $\begin{array}{l}\text { Demographic } \\
\text { characteristic }\end{array}$ & Details/subgroups & Number & Percentage \\
\hline \multirow{4}{*}{ Age } & $<5$ Years & 73 & $34.1 \%$ \\
\hline & 6-10 Years & 71 & $33.2 \%$ \\
\hline & $11-15$ Years & 54 & $25.2 \%$ \\
\hline & $>15$ Years & 16 & $7.5 \%$ \\
\hline \multirow[t]{4}{*}{ Duration of fever } & $<7$ days & 139 & $65 \%$ \\
\hline & 8-14 days & 36 & $17 \%$ \\
\hline & $15-21$ days & 21 & $10 \%$ \\
\hline & $>21$ days & 18 & $8 \%$ \\
\hline \multirow[t]{4}{*}{ Vaccination Status } & Age appropriate & 190 & $89 \%$ \\
\hline & Incomplete & 9 & $4 \%$ \\
\hline & Unvaccinated & 6 & $3 \%$ \\
\hline & No information/history & 9 & $4 \%$ \\
\hline \multirow[t]{12}{*}{ Risk factors } & Undernutrition & 107 & $50 \%$ \\
\hline & Immunosuppression & 40 & $19 \%$ \\
\hline & Raw milk consumption & 4 & $1.7 \%$ \\
\hline & Contact with domestic animals & 4 & $1.7 \%$ \\
\hline & Insect bite & 22 & $10.3 \%$ \\
\hline & Immunosuppression + Raw milk & 4 & $1.7 \%$ \\
\hline & Outside food consumption & 7 & $3.4 \%$ \\
\hline & $\begin{array}{l}\text { Undernutrition }+ \text { Contact with } \\
\text { domestic animals }\end{array}$ & 4 & $1.7 \%$ \\
\hline & $\begin{array}{l}\text { Undernutrition }+ \\
\text { Immunosuppression }\end{array}$ & 22 & $10.3 \%$ \\
\hline & Past history of surgery & 14 & $6.5 \%$ \\
\hline & Chronic illness & 15 & $7 \%$ \\
\hline & Recent travel & 6 & $2.8 \%$ \\
\hline \multirow[t]{7}{*}{ Clinical symptoms } & Cough & 82 & $38.3 \%$ \\
\hline & Abdominal pain & 49 & $28.8 \%$ \\
\hline & Headache & 36 & $16.8 \%$ \\
\hline & Vomiting & 82 & $38.3 \%$ \\
\hline & Diarrhoea & 15 & $7 \%$ \\
\hline & Breathlessness & 7 & $3.2 \%$ \\
\hline & Burning micturition & 3 & $1.4 \%$ \\
\hline \multirow[t]{7}{*}{ Clinical signs } & Anaemia & 55 & $25.7 \%$ \\
\hline & Jaundice & 16 & $7.47 \%$ \\
\hline & Neck rigidity & 2 & $0.93 \%$ \\
\hline & Hepatomegaly & 70 & $33.1 \%$ \\
\hline & Splenomegaly & 19 & $8.87 \%$ \\
\hline & Crepitations & 7 & $3.27 \%$ \\
\hline & Rash & 30 & $14.4 \%$ \\
\hline
\end{tabular}


contact with domestic animals, $10.3 \%$ gave a history of recent insect bite and $3.4 \%$ of the patients consumed outside food.

Only $2.8 \%$ of the patients gave a positive history of recent travel. $88.8 \%$ of the children were vaccinated appropriately according to their age, whereas remaining were not vaccinated or had not completed their vaccination dose.

Other than fever, cough and vomiting were the most common symptoms accounting to $38.2 \%(82 / 214)$ of children. $22.8 \%$ and $16.8 \%$ of children experienced pain abdomen and headache, respectively. Most frequent clinical signs were hepatomegaly $(33.1 \%)$, anaemia $(25.7 \%)$ and rash (14.4\%).

$8.4 \%$ of the patients had thrombocytopenia, $18.9 \%$ had leukopenia, and $36.4 \%$ tested leukocytosis. $39.5 \%$ and $48.7 \%$ of patients had increased ALT and AST, respectively. $27.6 \%$ of children also tested hypoalbuminemia. Mean PT was $13.28 \mathrm{~s}$, while mean aPTT was $34.26 \mathrm{~s}$ in the study population [Table 2] [Fig. 1].

\subsection{Serological work-up}

Serological tests gave a positive result in $14.9 \%$ cases $(32 / 214)$. The most common serologically confirmed diagnosis was Dengue in 10 out of 94 tested samples $(10.6 \%)$ whereas percentage positivity was high in IgM ELISA for HAV (31.8\%, 7/22). Four patients each tested positive for IgM ELISA for Leptospirosis and Scrub typhus out of 79 and 135 tested, respectively. Most common serological investigation performed was IgM ELISA for Scrub typhus followed by Widal test [Fig. 2].

There were three cases with positive blood culture and one patient with a positive urine culture. The blood cultures isolated Methicillinresistant Staphylococcus aureus.

Duration of fever did not show statistical association with serological test positivity. Among the serological tests, positivity for IgM ELISA for HAV had significant statistical association with pain abdomen $(\mathrm{p}=$ 0.008 ) and hepatomegaly ( $\mathrm{p}=0.01$ ). Widal positivity was significantly associated with pain abdomen and diarrhoea $(\mathrm{p}=0.02$ and $\mathrm{p}=0.04)$. Positive history of breathlessness was associated with IgM ELISA for Scrub typhus with a $\mathrm{p}$ value of 0.04 . Dengue serology was significantly associated with hepatomegaly $(\mathrm{p}=0.001)$. Other clinical findings as anemia, clubbing, splenomegaly failed to statistically show association with serological positivity.

\subsection{Clinical outcome}

Clinical recovery was seen in 207 cases accounting for a recovery rate of $96.5 \%$. Two cases were discharged against medical advice and one child expired due to sepsis. We could not get outcome result for two cases. Serologically positive Dengue cases showed a recovery of $90 \%$. For the other serological tests, all positive cases showed recovery. There was no statistical significance established between test positivity and clinical outcome.

Table 2

Statistics from laboratory parameters.

\begin{tabular}{lll}
\hline Laboratory parameter & Median & Inter quartile range \\
\hline Haemoglobin $(\mathrm{g} / \mathrm{dl})$ & 11.8 & $(10.5,11.8)$ \\
Total WBC $\left(\right.$ cells $\left./ \mathrm{mm}^{3}\right)$ & 8750 & $(5,500,13,675)$ \\
Platelets $\left(\right.$ cells $\left./ \mathrm{mm}^{3}\right)$ & $2,35,000$ & $(1,39,000,3,29,750)$ \\
Total bilirubin $(\mathrm{mg} / \mathrm{dl})$ & 0.41 & $(0.23,1.20)$ \\
Conjugated bilirubin $(\mathrm{mg} / \mathrm{dl})$ & 0.16 & $(0.11,0.51)$ \\
Unconjugated bilirubin $(\mathrm{mg} / \mathrm{dl})$ & 0.22 & $(0.11,0.41)$ \\
AST $(\mathrm{u} / \mathrm{L})$ & 43 & $(32,102)$ \\
ALT $(\mathrm{u} / \mathrm{L})$ & 26 & $(15.25,74.75)$ \\
ALP $(\mathrm{IU} / \mathrm{L})$ & 163.5 & $(125.25,227.50)$ \\
Serum albumin $(\mathrm{g} / \mathrm{dl})$ & 3.8 & $(3.3,4.8)$ \\
\hline
\end{tabular}

\section{Discussion}

The study is one of only a few in the world that focusses solely on pediatric patients. It characterizes children presenting to tertiary healthcare with AFI in South India. A total 214 pediatric patients with AFI symptoms could be evaluated as part of the study.

Most of our study population consisted of children $<5$ years of age (34.1\%). Common organ systems involved in our study were upper respiratory tract and gastrointestinal tract. However, in $64 \%$ of the patients, fever was the only presenting symptom. According to a survey by Kheng Chheng et al., ${ }^{8}$ Lower respiratory tract infection (LRTI) and gastroenteritis were the most common findings. Majority of the patients presented with cough and vomiting, followed by pain abdomen and headache. Most patients showed hepatomegaly, followed by anaemia and rash. Hepatomegaly also was significantly associated with positivity rate of IgM ELISA for HAV. Anaemia observed in many cases might be due to its association with acute infections. In viral infections, it might be due to inhibition of medullary erythropoiesis. ${ }^{9}$ In children, however, the degree of anaemia depends on whether the child has concomitant haemolytic anaemia or is healthy-in which case, the drop in haemoglobin is, slow and there is the rapid recovery of RBCs in one to two weeks. According to a study by Marcos et al., ${ }^{10}$ children aged six months5 years, are particularly prone to iron deficiency anaemia.

The study also showed thrombocytopenia in $8.4 \%$ and leukocytosis in $36.4 \%$ of patients. Febrile thrombocytopenia is seen in Malaria, Dengue and Enteric fever, which is relevant to the obtained findings. Leukocytosis, on the other hand, is almost always present along with acute infections. Liver function tests reveal elevated liver enzymes like ALT (40\%), AST (49\%) and ALP (62\%). Most of the causative agents of AFI are known to affect liver functions.

Dengue was the common infectious aetiology followed by Hepatitis A and Scrub typhus as per serology. The review by Iroh Tam PY et al., ${ }^{11}$ Leptospirosis, Dengue, Rickettsiosis and Typhoid are the common diseases in the Asian region.

In a study conducted by Yogendra Pandurang Shelke et al., ${ }^{12}$ Scrub typhus was the predominant (47\%) infection among the AFI cases, followed by Dengue $17.40 \%$ and malaria in $12 \%$ cases. A study conducted by Jung et al. ${ }^{13}$ showed $56.6 \%$, and a study conducted by Sinha et al. ${ }^{14}$ did not show scrub typhus patients with eschar among 42 positive cases of scrub typhus which was included in their research. Our study results were like the study conducted by Solanke et al., ${ }^{15}$ in which Dengue positivity was $28.4 \%$. Along with serology, they had also detected Dengue by PCR. In India, dengue positivity ranged between $8 \%$ and $71 \%$ among AFI cases. The studies conducted in Tamil Nadu, Dehradun, showed Dengue is a prime cause of AFI cases. We reported Leptospirosis in $2 \%$ of AFI cases, like study carried out by Joshi et al. and Yogendra Pandurang Shelke et al. ${ }^{4,12}$

The clinical recovery was found to be $96.5 \%$ in present study. In a study from Africa conducted by Kabore et al., ${ }^{16} 85.1 \%$ clinically recovered, $9.2 \%$ were referred to other centres, $1.5 \%$ were sent home against medical advice and $4.2 \%$ died. In another study conducted by Chheng et al. ${ }^{8}$ the median duration between acute and discharge samples was 4 days and a mortality of $5.6 \%$ was observed, mostly in children with comorbidities. In a Singapore based study ${ }^{17}$ the recovery rate was at an average of 1.18 cases per person-week. Also, those with low WBC count had slower recovery as compared to those with normal or higher WBC count.

Our study reinforces that most of the clinical management relies on assessing clinical signs and symptoms compared to microbiological investigations. It also shows the necessity to have a diagnostic algorithm that includes all testing modalities depending on illness and presentation time. The study also gives an idea about the crucial aspects to be necessarily considered for the definitive diagnosis. We also agree that the study had shortcomings in terms of sample size. We could not establish a statistical association between duration of fever and test positivity. This would have increased the strength of serological 


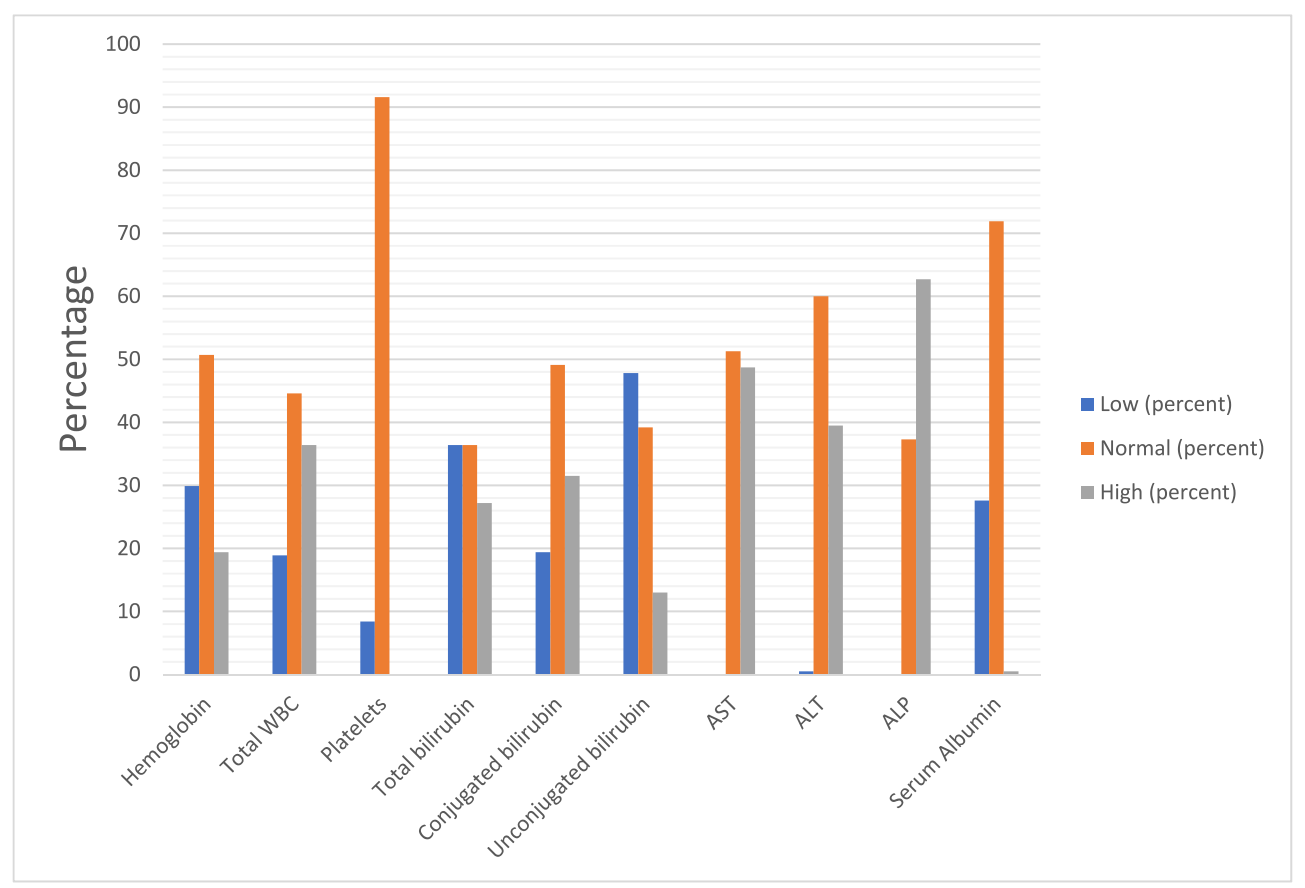

Fig. 1. Laboratory parameters of pediatric patients suffering from AFI.

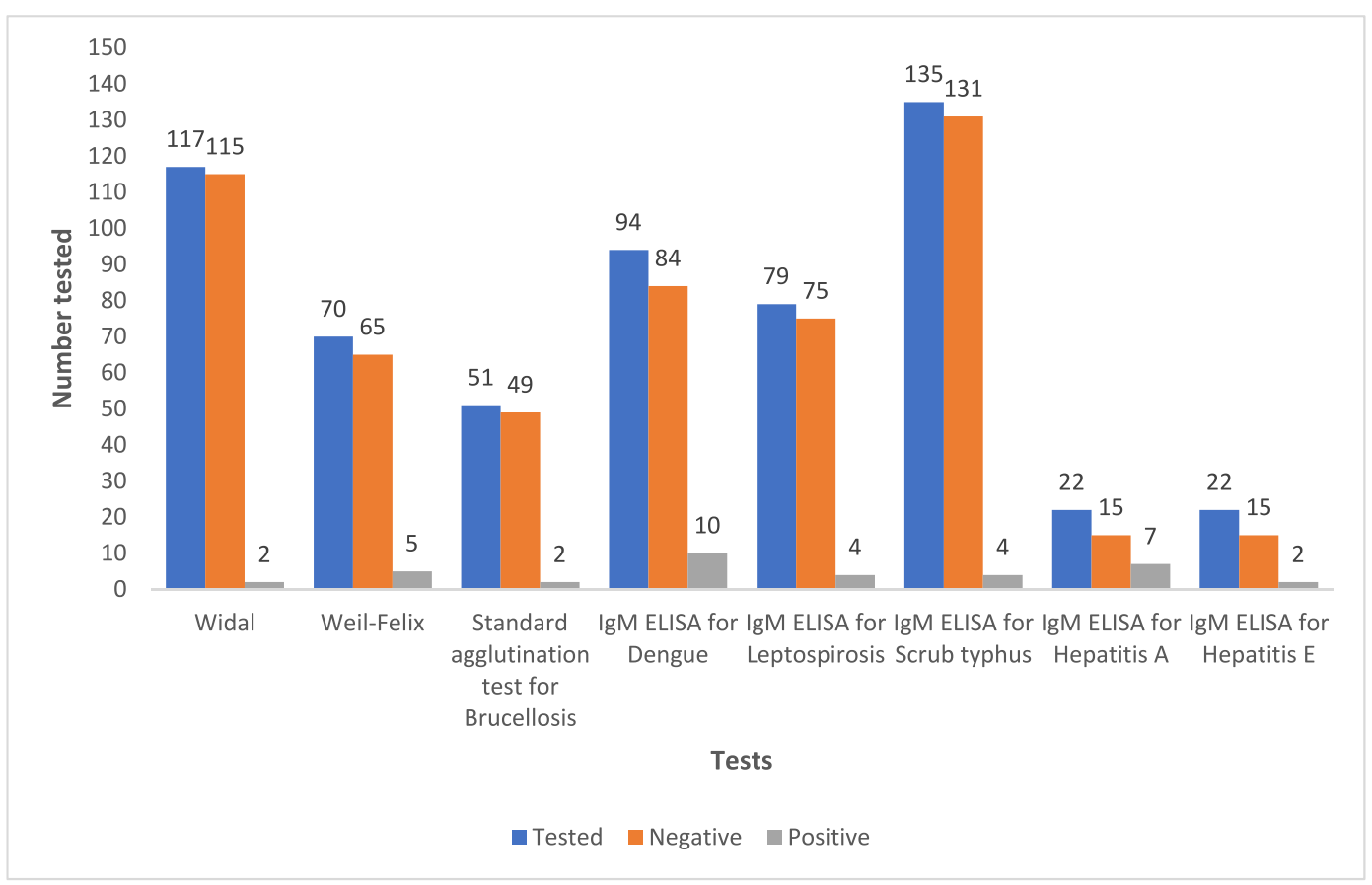

Fig. 2. Serological test results in pediatric patients suffering from AFI.

evidence in diagnosis of pediatric AFI.

A larger cohort would have helped to look at the diagnostic role of each of the serological assays used. We also could have compared cases with a definitive diagnosis with those without a laboratory-confirmed diagnosis. However, due to the smaller sample and retrospective study design, statistical outcome could not be strong in the study.

\section{Conclusion}

The diagnosis and management of Acute Febrile Illness continues to pose as a concern to the physician. Even with all the newer diagnostic facilities, AFI remains a challenge for clinical decision making. The limited number of studies conducted on pediatric patients with AFI makes it difficult to compare and contrast with various aetiologies, diagnostic modalities, treatment measures and post discharge recovery rates across the world. Overlapping clinical signs and symptoms and cross-reacting results of the serological tests are the main reasons for the same. Various diagnostic tests available target molecules which signify different stages of disease progression. Serology has been an essential component of the diagnostic algorithm. Though popular view is to evaluate a test using the positivity that it offers in suspected case, it is also important look at the predictive power of negative result. Therefore, 
it is essential to develop a comprehensive approach towards diagnosis of pediatric AFI, that takes into consideration stage of illness, probable aetiology and diagnostic probabilities of each test.

\section{Ethics declaration}

Ethical approval was taken from the Institutional Ethics Committee (IEC) of the affiliated institution. Institutional ethics approval numberIEC 505-2019.

\section{Author contributions}

VK: Conceptualization, data collection, formal analysis and writing original draft. MM: Conceptualization, methodology design, supervision, writing-review, and editing, supervision. SC: data collection and formal analysis, SA: Conceptualization methodology design, writingreview, and editing, supervision. All the authors discussed the results and approved the final manuscript.

\section{Declaration of competing interest}

Authors declare that they have no conflict of interest.

The manuscript is an original work by the authors and has not been published anywhere else.

\section{Acknowledgements}

Funding: Nil.

\section{References}

1 Susilawati TN, McBride WJ. Southeast Asian J Trop Med Publ Health. 2014;45(3): 719-726.
2 Manocha H, Ghoshal U, Singh SK, Kishore J, Ayyagari A. J Assoc Phys India. 2004;52: 623-625.

3 Singh, et al. A study on common etiologies of acute febrile illness detectable by microbiological tests in a tertiary care hospital. Int.J.Curr.Microbiol.App.Sci. 2016;5 (7):670-674.

4 Joshi R, Colford Jr JM, Reingold AL, Kalantri S. Nonmalarial acute undifferentiated fever in a rural hospital in central India: diagnostic uncertainty and overtreatment with antimalarial agents. Am J Trop Med Hyg. 2008;78:393-399.

5 Murdoch DR, Woods CW, Zimmerman MD, et al. The etiology of febrile illness in adults presenting to Patan hospital in Kathmandu, Nepal. Am J Trop Med Hyg. 2004; 70:670-675.

6 Naing Cho, Mohd Kassim Ani Izzuani Binti. Scaling-up attention to nonmalaria acute undifferentiated fever. Trans R Soc Trop Med Hyg. 2012;106(6):331-332.

7 Al-Eissa YA, Ghazal SS, Al-Zamil FA, Al-Salloum AA, Al-Omair AO, Al-Nasser MN. Pattern of febrile illnesses in children seen at a pediatric ambulatory care setting. J Family Community Med. 2000;7(2):61-65.

8 Chheng K, et al. A prospective study of the causes of febrile illness requiring hospitalization in children in Cambodia. PLoS One. 2013;8, e60634.

9 Capeding MR, Chua MN, Hadinegoro SR, Hussain II , Nallusamy R, et al. Dengue and other common causes of acute febrile illness in Asia: an active surveillance study in children. PLoS Neglected Trop Dis. 2013;25(7), e2331, 7.

10 Viana MB. Anemia and infection: a complex relationship. Rev Bras Hematol Hemoter. 2011;33(2):90-92.

11 Iroh Tam PY, Obaro SK, Storch G. Challenges in the etiology and diagnosis of acute febrile illness in children in low- and middle-income countries. J Pediatric Infect Dis Soc. 2016;5(2):190-205.

12 Shelke YP, Deotale VS, Maraskolhe DL. Spectrum of infections in acute febrile illness in central India. Indian J Med Microbiol. 2017;35(4):480-484.

13 Jung HC, Chon SB, Oh WS, Lee DH, Lee HJ. Etiologies of acute undifferentiated fever and clinical prediction of scrub typhus in a non-tropical endemic area. Am J Trop Med Hyg. 2015;92:256-261.

14 Sinha P, Gupta S, Dawra R, Rijhawan P. Recent outbreak of scrub typhus in North Western part of India. Indian J Med Microbiol. 2014;32:247-250.

15 Solanke VN, Karmarkar MG, Mehta PR. Early dengue diagnosis: role of Rapid NS1 antigen, NS1 early ELISA, and PCR assay. Trop J Med Res. 2015;18:95-99.

16 Kaboré B, Post A, Lompo P, et al. Aetiology of acute febrile illness in children in a high malaria transmission area in West Africa. Clin Microbiol Infect. 2021;27(4): 590-596.

17 Tun ZM, Moorthy M, Linster M, et al. Characteristics of acute febrile illness and determinants of illness recovery among patients presenting to Singapore primary care clinics. BMC Infect Dis. 2016;16:612. 